\title{
Second Zagreb Index of Trees with Fixed Diameter
}

\author{
E. H. Zogić, E. R. Glogić
}

\begin{abstract}
Let $G$ be a simple graph with vertex set $V=V(G)=\left\{v_{1}, v_{2}, \ldots, v_{n}\right\}$ and edge set $E=E(G)$. For $v_{i} \in V(G)$, by $d_{i}=d_{i}(G)$ we denote the degree (number of neighbors) of the vertex $v_{i}$. The second Zagreb index is defined as $M_{2}(G)=\sum_{v_{i} v_{j} \in E(G)} d_{i} d_{j}$. In this paper, we study the minimal and maximal second Zagreb index of trees with fixed diameter.
\end{abstract}

Keywords: second Zagreb index, diameter, trees

\section{Introduction}

We recall some terminologies in graph theory from [1]. Let $G$ be a simple graph with vertex set $V=V(G)=\left\{v_{1}, v_{2}, \ldots, v_{n}\right\}$ and edge set $E=E(G)$. For $v_{i} \in V(G)$, by $d_{i}=d_{i}(G)$ we denote the degree (number of neighbors) of the vertex $v_{i}$. For any two distinct vertices $u$ and $v$ in $G$, the distance between $u$ and $v$, denoted by $d_{G}(u, v)$, is the number of edges in a shortest path joining $u$ and $v$. The diameter $d$ of $G$ is the maximum distance between any two vertices of $G$. Let $S_{n}$ and $P_{n}$ be a star and a path on $n$ vertices, respectively.

The second Zagreb index is defined as

$$
M_{2}(G)=\sum_{v_{i} v_{j} \in E(G)} d_{i} d_{j}
$$

The second Zagreb index is one of the oldest vertex-degree-based molecular structure descriptors, invented in the 1970s [9, 10]. Details of its theory can be found in the recent review [2] and the recent papers [5, 3, 4] whereas data on their history in [8].

Researching in the field of vertex-degree-based topological indices with given diameter is one of the attractive topics in the chemical graph theory. Well known Wiener index of a graph $G$ is defined by

$$
W(G)=\sum_{v_{i} v_{j} \in E(G)} d\left(v_{i}, v_{j}\right) .
$$

Manuscript received January 25, 2020. ; accepted March 26,2020.

E. H. Zogić and E. R. Glogić are with the State University of Novi Pazar, Department of Mathematical Sciences, Novi Pazar, Serbia 
Recently, Sun et al. in the paper [11] provides some new results about maximal values of $W(G)$ with given diameter. In the paper [13], Liu and Pan obtained some results on Wiener index of trees with fixed diameter.

The Hararay index of a graph $G$ is defined as

$$
H(G)=\sum_{\{u, v\} \subset V(G)} \frac{1}{d(u, v)} .
$$

In the the paper [6], Feng et al. obtained trees with minimal value of $H(G)$ with fixed diameter 3 and 4 .

Inspired by the paper [6] we consider second Zagreb index of trees which have minimal and maximal values with fixed diameter 3 and 4 . We will also compare our results with the upper bound from [12]

$$
M_{2}(G) \leq \frac{1}{2}\left(m^{2}-m(d-3)+(d-2)\right) \sqrt{2 m-n+1} .
$$

\section{Main results}

Let $\mathscr{T}_{n, d}$ be the set of trees of order $n$ and diameter $d$. In the class $\mathscr{T}_{n, 2}$ there is only one graph which is star $S_{n}$. The trees in $\mathscr{T}_{n, 3}$ are obtained from a path $P_{4}=v_{1} v_{2} v_{3} v_{4}$ by attaching $a$ pendant vertices at $v_{2}$ and $b$ pendant vertices at $v_{3}$. We will denote this tree by $T_{3}(a, b)$ (Figure 1).

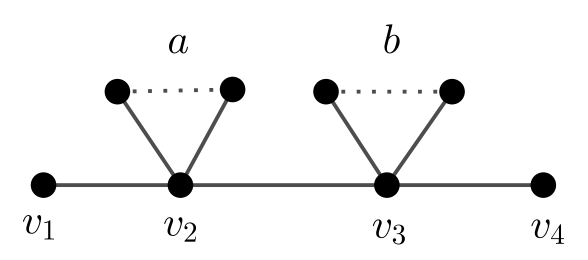

Fig. 1. $T_{3}(a, b)$

Theorem 2.1. For $T_{3}(a, b) \in \mathscr{T}_{n, 3}$, we have

$$
M_{2}\left(T_{3}(a, b)\right) \geq \begin{cases}\frac{3}{4} n^{2}-n, & \text { if } n \text { is even } \\ \frac{3}{4} n^{2}-n+\frac{1}{4}, & \text { if } n \text { is odd } .\end{cases}
$$

The equality holds if and only if $a=\frac{n-4}{2}$ when $n$ is even or $a=\frac{n-3}{2}$ or $a=\frac{n-5}{2}$ when $n$ is odd. 
Proof. Bearing in mind that $a+b=n-4$ and using Figure 1, we have

$$
\begin{aligned}
M_{2}\left(T_{3}(a, b)\right) & =a+2+a(a+2)+(a+2)(b+2)+b(b+2)+b+2 \\
& =5(a+b)+(a+b)^{2}-a b+8 \\
& =a^{2}-(n-4) a+n^{2}-3 n+4
\end{aligned}
$$

It follows that

$$
M_{2}\left(T_{3}(a, b)\right)=\left(a-\frac{n-4}{2}\right)^{2}-\frac{(n-4)^{2}}{4}+n^{2}-3 n+4
$$

If $n$ is even, then the minimal value of (2) is attained for $a=\frac{n-4}{2}$ and we have

$$
M_{2}\left(T_{3}(a, b)\right) \geq \frac{3}{4} n^{2}-n
$$

If $n$ is odd, then the minimal value of (2) is attained for $a=\frac{n-3}{2}$ or $a=\frac{n-5}{2}$ and we get

$$
M_{2}\left(T_{3}(a, b)\right) \geq \frac{3}{4} n^{2}-n+\frac{1}{4} .
$$

Now, we are going to find trees with maximal value of $M_{2}$ in the class $\mathscr{T}_{n, 3}$.

Theorem 2.2. (a) If $b \geq a$, then $M_{2}\left(T_{3}(a, b)\right)<M_{2}\left(T_{3}(a-1, b+1)\right)$.

(b) If $b<a$, then $M_{2}\left(T_{3}(a, b)\right)<M_{2}\left(T_{3}(a+1, b-1)\right)$.

Proof. Claims (a) and (b) follow from (2) and

$$
\begin{aligned}
& M_{2}\left(T_{3}(a-1, b+1)\right)=a^{2}+b^{2}+a b+4 a+6 b+9, \\
& M_{2}\left(T_{3}(a+1, b-1)\right)=a^{2}+b^{2}+a b+6 a+4 b+9 .
\end{aligned}
$$

Remark 2.1. By the theorem 2.2, for $T \in \mathscr{T}_{n, 3}$ it follows

$$
M_{2}(T) \leq n^{2}-3 n+4,
$$

with equality if and only if $T \cong T_{3}(0, n-4)$ or $T \cong T_{3}(n-4,0)$.

Remark 2.2. For $d=3$ and $m=n-1$ in (1) we obtain

$$
M_{2}(G) \leq \frac{1}{2}\left(n^{2}-2 n+2\right) \sqrt{n-1}
$$

The upper bound (3) is better than the upper bound (4). 


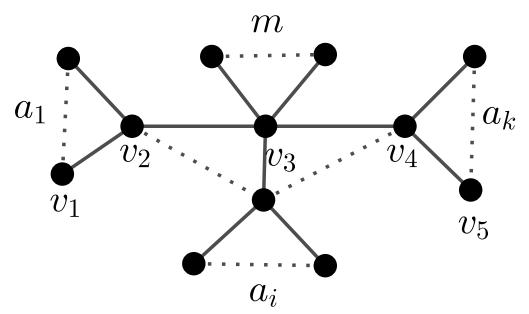

Fig. 2. $T_{4}\left(m ; a_{1}, a_{2}, \ldots, a_{k}\right)$

Next, we consider the trees with diameter 4 . Let $\mathscr{T}_{n, 4}$ be a class of trees with $n$ vertices and diameter $d=4$. The trees in $\mathscr{T}_{n, 4}$ are of the form like $T_{4}\left(m ; a_{1}, a_{2}, \ldots, a_{k}\right)$ with $m \geq 0$, as shown in figure 2 , where $\sum_{i=1}^{k} a_{i}+m+k+1=n$. The tree $T_{4}\left(m ; a_{1}, a_{2}, \ldots, a_{k}\right)$ is obtained from a path $P_{5}=v_{1} v_{2} v_{3} v_{4} v_{5}$ by attaching some pendant edges at $v_{2}, v_{4}$, and/or attaching some pendant edges at $v_{3}$, and/or identifying $v_{3}$ with a pendant vertex of a star. If $m=0$ we will write $T_{4}\left(a_{1}, a_{2}, \ldots, a_{k}\right)$ instead $T_{4}\left(m ; a_{1}, a_{2}, \ldots, a_{k}\right)$.

Theorem 2.3. For any tree of order $n \geq 16$ of the form $\tilde{T}=T_{4}\left(m ; a_{1}, a_{2}, \ldots, a_{k}\right) \in \mathscr{T}_{n, 4}$, where $k \geq 2, m \geq 1$, there exists a tree of order $n \geq 16$ of the form $T=T_{4}\left(b_{1}, b_{2}, \ldots, b_{t}\right)$ such that $M_{2}(\tilde{T}) \geq M_{2}(T)$.

Proof. Let $k \geq 2, m \geq 1$ and without loss of generality we assume $a_{1} \leq a_{2} \leq \cdots \leq a_{k}$. Let $T^{\prime}=T\left(m-1 ; a_{1}+1, a_{2}, \ldots, a_{k}\right)$. Now we have

$$
\begin{aligned}
M_{2}(\tilde{T}) & =\sum_{i=1}^{k}(m+3)\left(a_{i}+1\right)+\sum_{i=1}^{k} a_{i}\left(a_{i}+1\right)+m(m+3) \\
M_{2}\left(T^{\prime}\right)= & \sum_{i=2}^{k}(m+2)\left(a_{i}+1\right)+\left(a_{1}+2\right)(m+2) \\
& +\sum_{i=2}^{k} a_{i}\left(a_{i}+1\right)+\left(a_{1}+1\right)\left(a_{1}+2\right)+(m-1)(m+2) \\
= & (m+2) \sum_{i=2}^{k}\left(a_{i}+1\right)+(m+2)\left(a_{1}+1\right)+\sum_{i=1}^{k}\left(a_{i}+1\right)+\sum_{i=2}^{k} a_{i}\left(a_{i}+1\right) \\
M_{2}(\tilde{T})-M_{2}\left(T^{\prime}\right) & a_{1}\left(a_{1}+1\right)+m(m+3) \\
- & (m+2) \sum_{i=2}^{k}\left(a_{i}+1\right)-\left(a_{1}+2\right)(m+2)-\sum_{i=2}^{k} a_{i}\left(a_{i}+1\right) \\
- & \left(a_{1}+1\right)\left(a_{1}+2\right)-(m-1)(m+2) \\
= & \sum_{i=1}^{k} a_{i}+k+m-2 a_{1}-2 \geq 0 .
\end{aligned}
$$


The last inequality holds because $k \geq 2, \sum_{i=1}^{k} a_{i} \geq k a_{1} \geq 2 a_{1}$ and $m \geq 1>0$.

Remark 2.3. From the Theorem 2.3, one can see that tree with minimal value of $M_{2}$ is of the form $T_{4}\left(a_{1}, a_{2}, \ldots, a_{k}\right)$ and it holds that $n-1-k \geq k$, i.e., $k \leq \frac{n-1}{2}$.

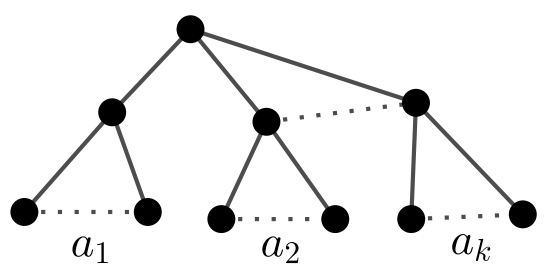

Fig. 3. $T_{4}\left(a_{1}, a_{2}, \ldots, a_{k}\right)$

Theorem 2.4. Let $T=T_{4}\left(a_{1}, a_{2}, \ldots, a_{k}\right)$ be the tree of order $n$ with diameter 4 and $k \geq 2$ as depicted in Figure 3. Then we have

$$
M_{2}(T)=(n-1) k+n-1 .
$$

Proof. Since $m=0$ and $\sum_{i=1}^{k} a_{i}=n-k-1$, we have

$$
M_{2}(T)=\sum_{i=1}^{k}\left(a_{i}+1\right)+\sum_{i=1}^{k} k\left(a_{i}+1\right)=(k+1) \sum_{i=1}^{k}\left(a_{i}+1\right)=(n-1) k+n-1 .
$$

Remark 2.4. Since $k \geq 2$, one can see that (7) is minimized for $k=2$. By theorems 2.3 and 2.4, for $T \in \mathscr{T}_{n, 4}$ it follows that

$$
M_{2}(T) \geq M_{2}\left(T\left(a_{1}, a_{2}, 0, \ldots, 0\right)\right) .
$$

Next, we consider the trees form the class $\mathscr{T}_{n, 4}$ which have maximal value of $M_{2}$.

Theorem 2.5. $M_{2}\left(T_{4}\left(m ; a_{1}, a_{2}, \ldots, a_{k}\right)\right)<M_{2}\left(T_{4}\left(m+1 ; a_{1}-1, a_{2}, \ldots, a_{k}\right)\right)$.

Proof. Using the right-hand side of (5) and

$$
\begin{aligned}
M_{2}\left(T_{4}\left(m+1 ; a_{1}-1, a_{2}, \ldots, a_{k}\right)\right) & =\sum_{i=2}^{k}(m+4)\left(a_{i}+1\right)+(m+4) a_{1} \\
& +\sum_{i=2}^{k} a_{i}\left(a_{i}+1\right)+\left(a_{1}-1\right) \cdot a_{1}+(m+1)(m+4),
\end{aligned}
$$

we have

$$
M_{2}\left(T_{4}\left(m+1 ; a_{1}-1, a_{2}, \ldots, a_{k}\right)\right)-M_{2}\left(T_{4}\left(m ; a_{1}, a_{2}, \ldots, a_{k}\right)>0 .\right.
$$


Remark 2.5. By the Theorem 2.5 one can see that for $T \in \mathscr{T}_{n, 4}$ we have

$$
M_{2}(T) \leq n^{2}-4 n+7,
$$

with equality if and only if $T \cong T_{4}(n-k-3 ; 1,0,0, \ldots, 1)$. Notice that

$$
\begin{aligned}
M_{2}\left(T_{4}(n-k-3 ; 1,0,0, \ldots, 0,1)\right) & =M_{2}\left(T_{4}(n-k-3 ; 0,1,0, \ldots, 0,1)\right) \\
& =\cdots=M_{2}\left(T_{4}(n-k-3 ; 0,0,0, \ldots, 1,1)\right) .
\end{aligned}
$$

Remark 2.6. For $d=4$ and $m=n-1$ in (1) we obtain

$$
M_{2}(G) \leq \frac{1}{2}(n-1)(n-2) \sqrt{n-1}+\sqrt{n-1} .
$$

The upper bound (8) is better than the upper bound (9).

\section{References}

[1] C. Berge, Graphs, North-Holland, Amsterdam, 1985.

[2] B. Borovićanin, B. Furtula, K. C. Das, I. Gutman, Zagreb indices: bounds and extremal graphs, in: I. Gutman, B. Furtula, K. C. Das, I. Ž Milovanović, E. I. Milovanović, Bounds in Chemical Graph Theory - Basics, Univ. Kragujevac, Kragujevac, 2017, pp. 67-153.

[3] B. BorovićAnin, T. Aleksić LAMPert, On the Maximum and Minimum Zagreb Indices of Trees with a Given Number of Vertices of Maximum Degree, MATCH Commun. Math. Comput. Chem. 74 (2015), 81-96.

[4] B. Borovićanin, On the Extremal Zagreb Indices of Trees with Given Number of Segments or Given Number of Branching Vertices, MATCH Commun. Math. Comput. Chem. 74 (2015), 57-79.

[5] K. C. Das, K. Xu, I. Gutman, On Zagreb and Harary indices, MATCH Commun. Math. Comput. Chem. 70 (2013), 301-314.

[6] L. Feng, Y. LAN, W. LiU, X. WAng, Minimal Harary Index of Graphs with Small Parameters, MATCH Commun. Math. Comput. Chem. 76 (2016), 23-42.

[7] I. Gutman, Graphs with smallest sum of squares of vertex degrees, Kragujevac J. Math. 25 (2003), 51-54.

[8] I. Gutman, On the origin of two degree-based topological indices, Bull. Acad. Serbe Sci. Arts (Cl. Sci. Math. Natur.) 146 (2014), 39-52.

[9] I. Gutman, B. Ruščić, N. Trinajstić, C. F. Wilcox, Graph theory and molecular orbitals. XII. Acyclic polyenes, J. Chem. Phys. 62 (1975), 3399-3405.

[10] I. Gutman, N. Trinajstić, Graph theory and molecular orbitals. Total $\pi$-electron energy of alternant hydrocarbon, Chem. Phys. Lett. 17 (1972), 535-538.

[11] Q. Sun, B. IKICA, R. ŠKReKovSKI, V. VukašINović, Graphs with a given diameter that maximise the Wiener index, Appl. Math. Comput. 356 (2019), 438-448. 
[12] B. LiU, I. GUTMAN, Upper bounds for Zagreb indices of connected graphs, MATCH Commun. Math. Comput. Chem. 55 (2006), 439-446.

[13] H. LIU, X. PAN, On the Wiener Index of Trees with Fixed Diameter, MATCH Commun. Math. Comput. Chem. 60 (2008), 85-94. 